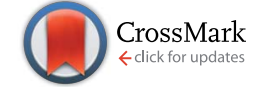

Cite this: RSC Adv., 2017, 7, 4011

Received 8th November 2016

Accepted 24th December 2016

DOI: $10.1039 / \mathrm{c} 6 \mathrm{ra2} 6500 \mathrm{f}$

www.rsc.org/advances

\section{Simultaneous electrokinetic concentration and separation of proteins on a paper-based analytical device $\uparrow$}

\author{
Zhi-Yong Wu, ${ }^{* a}$ Biao Ma, ${ }^{a}$ Song-Fang Xie, ${ }^{a}$ Kun Liu ${ }^{b}$ and Fang Fang ${ }^{a}$
}

In this report, we introduced an electrokinetic strategy for simultaneous concentration and separation of charged analytes in an open and free standing paper fluidic channel. This was achieved by taking advantage of the field amplified sample stacking (FASS) effect. An electric field gradient was developed in the channel due to the conductivity difference between the background electrolyte (BGE) and sample solution. Through adjustment of the electroosmotic flow level and BGE concentration, charged analytes were not only stacked but also separated in the gradient based on their difference in electrophoretic mobility. Proof of concept was demonstrated firstly by two food dyes, brilliant blue and amaranth, and each dye was about 100-fold concentrated within 300 s. Simultaneous stacking and separation of two colored proteins, bovine hemoglobin and cytochrome c, was also demonstrated visually with the proposed method. Sample consumption was reduced to $5 \mu \mathrm{L}$ by directly loading the sample onto the open channel, and the separation was completed within $30 \mathrm{~s}$. This method is simple, rapid and easy to operate, which is particularly beneficial to the development of sensitive and multifunctional paper-based analytical devices (PADs) for field testing.

\section{Introduction}

Paper substrates possess many advantages such as low cost, light weight, high surface area to volume ratio, capillary action, biocompatibility, flexibility and disposability. ${ }^{1,2}$ In 2007, paper as a substrate material for microfluidic devices was first reported by the Whitesides research group. ${ }^{3}$ Since then, the field of microfluidic paper-based analytical devices ( $\mu$ PADs) has experienced rapid growth due to their salient advantages such as easy fabrication, portability, low sample consumption, multiple assays and simple to operate. ${ }^{4-8}$ These advantages make $\mu$ PADs highly attractive for point of care testing (POCT), especially in resource-limited settings. To date, the application scope of $\mu$ PADs has involved medical diagnosis, ${ }^{9,10}$ environmental analysis, ${ }^{\mathbf{1 1}}$ food safety control ${ }^{12}$ and drug testing. ${ }^{13}$

Preconcentration and separation of sample components are essential steps in many analyses as well as medical diagnostic tests. Despite a number of assays have been demonstrated on

\footnotetext{
${ }^{a}$ Research Center for Analytical Sciences, Chemistry Department, College of Sciences, Northeastern University, Shenyang 110819, P. R. China. E-mail: zywu@mail.neu. edu.cn; Fax: +86 248368 7659; Tel: +86 2483687659

${ }^{b}$ Research Institute of Vacuum and Fluid, College of Mechanical Engineering and Automation, Northeastern University, Shenyang 110819, P. R. China

$\dagger$ Electronic supplementary information (ESI) available: The photo of the smartphone-based imaging system (Fig. S1), details about the construction of the calibration curves (Fig. S2), data processing of image (Fig. S3), the stacking and separation process for low sample consumption (Fig. S4). See DOI: 10.1039/c6ra26500f
}

$\mu \mathrm{PADs}$, the integration of multiple pretreatment units remains a challenge. A few efforts have been exerted to add preconcentration function on $\mu$ PADs, including liquid evaporation, ${ }^{\mathbf{1 4}}$ dialysis $^{15}$ and chemical modifications on the paper substrate. ${ }^{16,17}$ In addition, some electrokinetic preconcentration techniques developed in capillary electrophoresis and microfluidic system were also demonstrated on $\mu$ PADs. These techniques include ion concentration polarization (ICP), ${ }^{\mathbf{1 8 - 2 5}}$ isotachophoresis (ITP) ${ }^{\mathbf{2 6 - 2 9}}$ and field amplified sample stacking (FASS), ${ }^{30}$ which are easy to perform on $\mu$ PADs thanks to the openness of the paper fluidic channel and the simplicity in surface modification.

Paper electrophoresis is a very traditional separation method, and now it has been mostly replaced by slab gel or capillary electrophoresis. Recently, the potential function on electrophoretic separation is redrawing attention in the new scenario of $\mu$ PADs, as have been demonstrated for electrophoretic separation of amino acids, ${ }^{31}$ food dyes, ${ }^{32}$ metal ions, ${ }^{33}$ proteins $^{34,35}$ and nucleic acids. ${ }^{19}$ However, in most cases, the preconcentration and separation steps were performed independently that could not achieve the sensitive detection of complex and low abundant sample components. An applicable way to address this issue is through the combination of the sample preconcentration and separation in a single step. This may be realized by using the present electrokinetic techniques in microfluidic system, such as isoelectric focusing, ${ }^{36}$ ICP, ${ }^{37}$ ITP ${ }^{38}$ bipolar electrode focusing ${ }^{39}$ and conductivity gradient focusing. ${ }^{40}$ Among these techniques, only ICP was carried to $\mathrm{PADs}$ by Gong and co-workers. ${ }^{19}$ They demonstrated 
simultaneous preconcentration and separation of hepatitis B virus DNA fragments in human serum by ICP in a nitrocellulose paper fluidic channel. By local patterning of cation selective nanoporous membrane (Nafion) in the channel, an electric field gradient was induced under applied voltage. They also extended the paper-based ICP effect to preconcentrate and separate the ssDNA and dsDNA from lysed sperm cells in order to analyse the integrity of sperm DNA. ${ }^{41}$

FASS is believed as the simplest online preconcentration technique in capillary electrophoresis. ${ }^{\mathbf{4 2 - 4 4}}$ In our previous work, this technique was first introduced to a $\mu \mathrm{PAD}$ composed of glass fiber paper and up to 1000-fold enrichment factor was obtained. ${ }^{30}$ Inspired by the aforementioned work by Gong et al. ${ }^{19}$ we envisioned that the electric field gradient as developed in the paper-based FASS system should not only enable charged species to be stacked, but also have the potential to separate them based on their difference in electrophoretic mobility. Through the optimization of experimental conditions, simultaneous stacking and separation of small molecule food dyes was demonstrated in the paper fluidic channel, and about 100fold enrichment factor was obtained for each dye within $300 \mathrm{~s}$. Then we demonstrated concentration and separation of macromolecule proteins. This method is low cost, simple, rapid and easy to operate, which is particularly beneficial to the development of sensitive and multifunctional paper-based analytical devices (PADs) for field testing.

\section{Experimental}

\section{Chemicals and materials}

Tris(hydroxymethyl)aminomethane (Tris), ammonium chloride $\left(\mathrm{NH}_{4} \mathrm{Cl}\right.$ ), brilliant blue FCF (MW 792.85), amaranth (MW 604.47) and hydroxyethylcellulose (HEC) were purchased from Aladdin Reagent (Shanghai, China). Hydrochloric acid ( $\mathrm{HCl})$ and ammonia solution were purchased from Sinopharm Chemical Reagent Co., Ltd. Bovine hemoglobin (BHb, MW $65 \mathrm{kDa}, \mathrm{pI} \sim$ 7.0) and cytochrome c (Cyt c, from bovine heart, MW $\sim 13 \mathrm{kDa}, \mathrm{pI}$ $\sim$ 10.1) were obtained from Sangon Biotech (Shanghai, China). Deionized water was obtained from the Millipore Milli-Q system (Millipore, Milford, MA). $500 \mathrm{mM}$ Tris-HCl ( $\mathrm{pH}=8.1$ ) was prepared as the stock background electrolyte. Glass fiber paper (K49, $90 \mathrm{~mm}$ in diameter and $250 \mu \mathrm{m}$ in thickness) was purchased from Dezhou Kejia Environmental Products Co. The glass fiber paper was cut into strips ( $30 \mathrm{~mm}$ long by $3 \mathrm{~mm}$ wide) using a paper trimmer. The lids of the centrifuge tube $(1.5 \mathrm{~mL})$ were used as the reservoirs. Platinum wires ( $0.5 \mathrm{~mm}$ in diameter) were used as electrodes. A miniature and low cost DC-DC booster converter (GRB12300D, ESSO Latham Electronic Technology Co., Ltd., Shenzhen, China) linked with a USB portable battery power charger (10 400 mA h, 5 V output, PH50, Romoss Technology Co., Ltd., Shenzhen, China) was taken as the power supply. The booster converter gives a constant output voltage of $300 \mathrm{~V}$.

\section{Device operation}

The paper strip was placed on two reservoirs at a distance of $20 \mathrm{~mm}$. For each side of the strip, $5 \mathrm{~mm}$ was immersed into the solution. $150 \mu \mathrm{L}$ background electrolyte (BGE) was firstly loaded into the anode reservoir, and most of paper channel (from anode side to the edge of the cathode reservoir) was wetted using BGE. Then $150 \mu \mathrm{L}$ sample solution was loaded into the cathode reservoir, followed by applying the voltage.

\section{Detection and quantitation}

A smartphone (Meizu Note1, running Android 5.1) was fixed to the eyepiece tube of the stereomicroscope by an attaching clamp and the back camera was adjusted to align with the eyepiece. In order to avoid the interruption of the ambient light, the stereomicroscope was surrounded using a shade cloth. A white LED light was used to illuminate the paper strip to provide a stable light condition. The photo of the smartphonebased imaging system is shown in Fig. S1 (ESI $\dagger$ ). The smartphone was used with a professional camera control application (Camera FV-5 Lite, Version 3.15).

Images captured from the smartphone were processed and analyzed using ImageJ software (Version 1.49v, National Institutes of Health, Bethesda, MD). For quantitative assessment of the enrichment factor (EF), calibration curves were constructed based on the mean gray values of the standard solutions. The complete details about the construction of calibration curves were provided in Fig. S2 (ESI $\dagger$ ). For calculating the color intensity of the stacking band, the region of each band was selected by the color threshold tool of ImageJ. Then the mean gray value of the selected zone was measured as the color intensity for quantitative analysis, as can be seen in Fig. S3 (ESI†). Note that each band intensity was corrected by subtracting the mean gray value of the BGE zone near the band.

\section{Results and discussion}

\section{Simultaneous concentration and separation}

When a voltage is applied across a paper fluidic channel which connects with a high conductivity BGE and a low conductivity sample solution, an electric field gradient can be developed in the channel. Once charged molecules migrate into the gradient, they stack together because of the reduction in electromigration velocity. This is the central idea for the paper-based field amplified sample stacking (FASS). Previous work was mainly focused on the use of FASS to preconcentrate analytes. ${ }^{30}$ Here, it is reasonable to assume that with proper condition optimization, analytes with different mobilities should not only be stacked, but also be separated in the electric field gradient, as shown in Fig. 1a. This was experimentally proved with two food dyes (brilliant blue and amaranth) as shown in Fig. 1b. Both of the dyes are negatively charged in aqueous solution. Two narrow stacking bands are clearly separated as expected. The sequence of the two bands is in agreement with the mobilities of the two dyes. ${ }^{45}$ The dyes were prepared with low conductivity deionized water while $50 \mathrm{mM}$ Tris- $\mathrm{HCl}$ was taken as the BGE. To successfully obtain the simultaneous stacking and separation, both the level of electroosmotic flow (EOF) and that of the electric field gradient should be well optimized, as can be seen in the following section. 

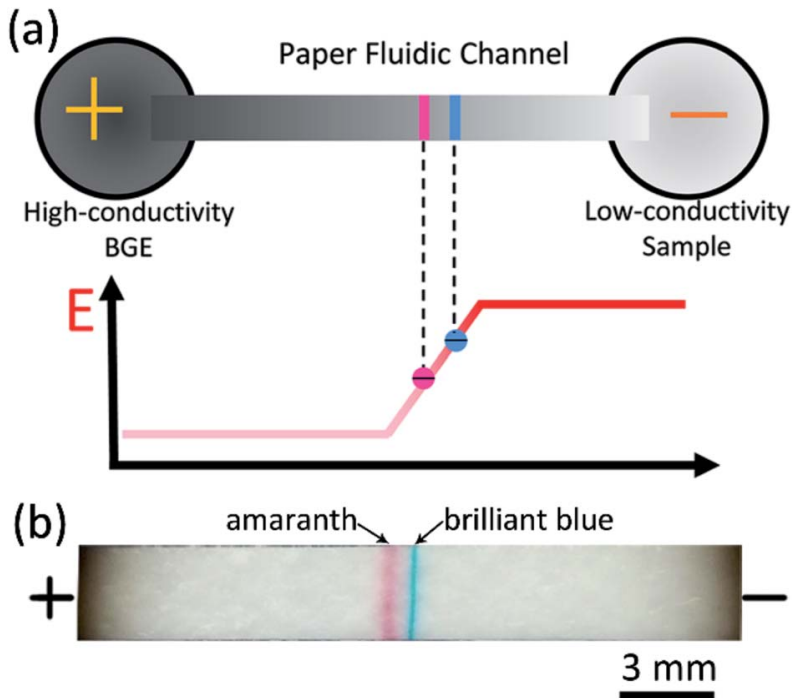

Fig. 1 (a) Schematic of paper-based FASS for simultaneous concentration and separation for negatively charged analytes. Stacking is achieved due to reduction of electric field, and separation is achieved because of the difference in electrophoretic mobility. (b) A typical image of the simultaneous concentration and separation of two negatively charged food dyes, brilliant blue and amaranth.

\section{Effect of EOF}

As is well known, the negatively charged surface of the glass fiber can generate an EOF in the direction of electric field. ${ }^{46}$ To investigate the effect of EOF level, BGE of $50 \mathrm{mM}$ Tris-HCl was maintained for each test. Fig. 2 shows the images of channel at representative time points of $30 \mathrm{~s}$ and $180 \mathrm{~s}$. Without the suppression of EOF (no HEC in BGE), neither stacking band nor separation was seen. This is because the EOF was too strong to develop a stable electric field gradient in the channel. Adding additive in the BGE is a convenient method to adjust the EOF level, as has been shown in the previous work where a surfactant of polyvinylpyrrolidone (PVP) was used. ${ }^{30}$ However, PVP was found not as effective for the two food dyes here, and the reason remained unknown to us. Here we used another EOF modifier, hydroxyethylcellulose (HEC). ${ }^{47}$ By adding $0.2 \%$ HEC $(\mathrm{m} / \mathrm{v})$ to the BGE, the two stacking bands emerged in the paper channel. When the HEC concentration was increased to $0.4 \%$, the bands became more concentrated and narrower, especially for the (a)

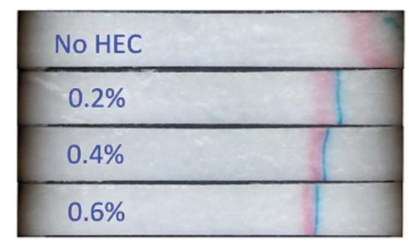

(b)

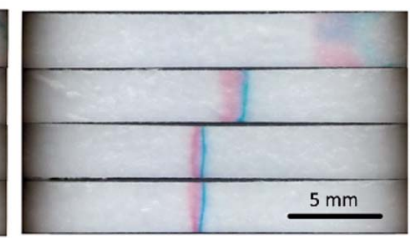

Fig. 2 Images of the stacking bands at $30 \mathrm{~s}$ (a) and $180 \mathrm{~s}$ (b) with addition of $\mathrm{HEC}$. BGE was $50 \mathrm{mM}$ Tris $-\mathrm{HCl}$, the initial concentration for each dye was $1.0 \mu \mathrm{g} \mathrm{mL}^{-1}$, voltage was $300 \mathrm{~V}$. The paper fluidic channel was $30 \mathrm{~mm}$ long by $3 \mathrm{~mm}$ wide. amaranth. It should be noted that HEC not only suppresses the EOF, but also increases the viscosity of the solution, which is beneficial to decrease the diffusion of the stacked molecules. When the HEC concentration was above $0.4 \%$, no obvious improvement was observed. Thus, 0.4\% HEC was added to each BGE in the following experiments.

\section{Effect of BGE concentration}

The conductivity difference between BGE and sample is another key factor for a successful FASS operation, which determines the profile of the electric field gradient. Tris-HCl buffers ranging from $25 \mathrm{mM}$ to $200 \mathrm{mM}$ were used to evaluate the effect of BGE concentration on the separation and concentration. As shown in Fig. 3a and b, when the Tris- $\mathrm{HCl}$ was $200 \mathrm{mM}$, a narrow stacking band formed in $30 \mathrm{~s}$, and kept well in $180 \mathrm{~s}$, with slow drifting toward the anode. This phenomenon is in agreement with what was observed in the previous work with fluorescent probes. However, the separation effect was hardly seen. This should be ascribed to that the electric field gradient was so sharp that the two dyes stacked together. With decrease of the Tris- $\mathrm{HCl}$ concentration from 200 to $25 \mathrm{mM}$, the two dyes were found well separated. Fig. 3c shows the intensity distributions along the paper fluidic channels at $180 \mathrm{~s}$, from which we can obtain the band peak intensity and the separation resolution (SR). The SR values were 1.4 and 0.9 for the BGE of 25 and $50 \mathrm{mM}$ Tris- $\mathrm{HCl}$, respectively. The lower the BGE concentration, the better the separation, which implies that the electric field gradient level can be tuned by the BGE concentration. Although the BGE of $25 \mathrm{mM}$ Tris- $\mathrm{HCl}$ gave a better SR,
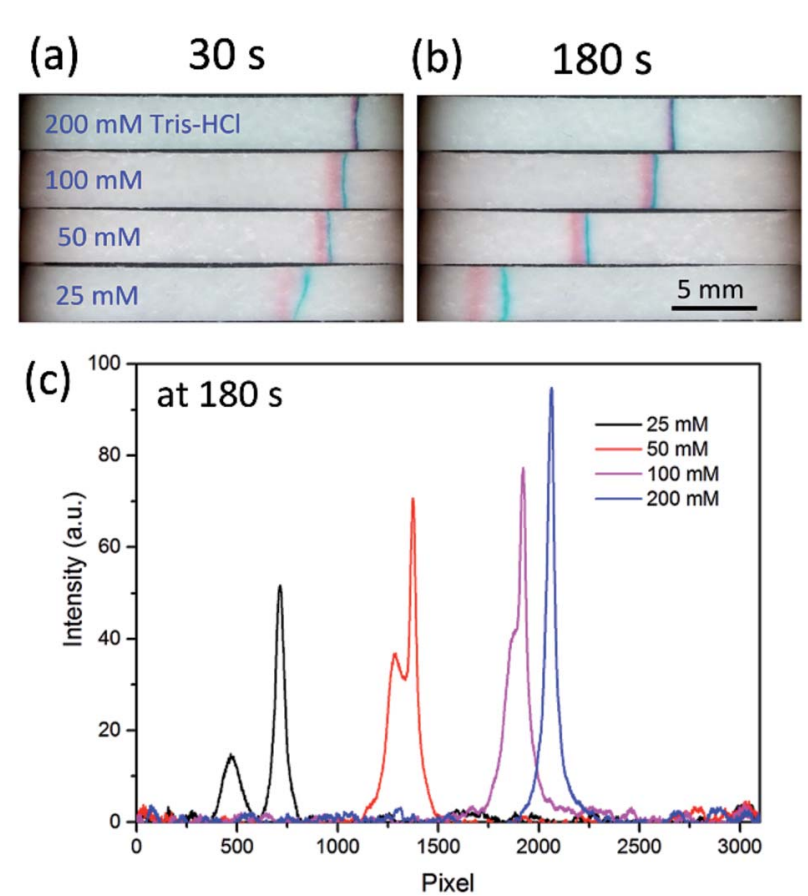

Fig. 3 Effect of the BGE (Tris- $\mathrm{HCl}$ ) concentration. Images of the stacking bands at $30 \mathrm{~s}$ (a) and $180 \mathrm{~s}$ (b), respectively. (c) The band intensity profile along the channel at $180 \mathrm{~s}$. The other conditions were the same as those in Fig. 2. 
the stacking efficiency is correspondingly reduced. Considering the compromise between separation performance and stacking efficiency, $50 \mathrm{mM}$ Tris-HCl was taken as the optimal BGE in the following section.

\section{Quantitative assessment}

Under the optimized conditions, quantitative assessment of the enrichment factor (EF) was performed based the images acquired by the smartphone-based imaging system. Fig. 4a depicts the band intensity as a function of time, and each intensity data was the averaged value of five parallel tests. The initial concentration for each dye was $1.0 \mu \mathrm{g} \mathrm{mL}{ }^{-1}$, and at this concentration each dye is invisible to naked eyes without stacking (not shown). To determine the EF values for the two dyes, calibration curves were established by measuring the intensities of standard solutions $\left(10-160 \mu \mathrm{g} \mathrm{mL}^{-1}\right)$, as shown in Fig. S2c and d (ESI $\dagger$ ). Without stacking, the limits of detection $(3 \sigma)$ for amaranth and brilliant blue were $21 \mu \mathrm{g} \mathrm{mL} \mathrm{m}^{-1}$ and $6.4 \mu \mathrm{g}$ $\mathrm{mL}^{-1}$, respectively. The EF values for amaranth and brilliant blue were 103 and 98, respectively, which were obtained by comparing the band intensity to the standard reference as indicated in Fig. 4a as dotted lines. Fig. 4b shows a typical stacking/separation process. Note that the light condition and the smartphone imaging parameters were kept constant for the acquired images.

To explore the potential of this method on quantitative analysis, the band intensity as a function of initial concentration under a given stacking/separation time was also investigated, as shown in Fig. 4c. The linear dynamic range for (a)

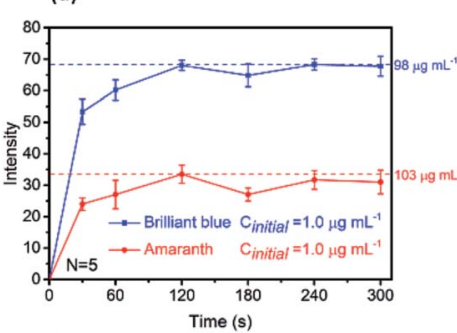

(c)

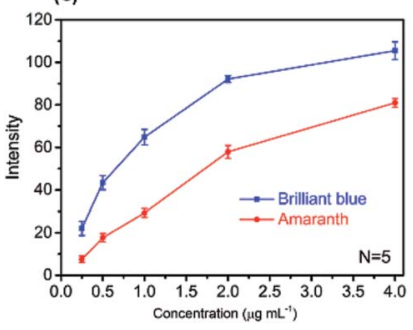

(b)

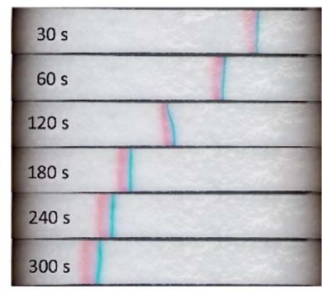

(d)

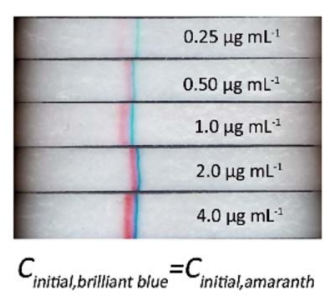

Fig. 4 Quantitation of paper-based FASS for sample concentration and separation. (a) Band's intensity development over time. The initial concentration for each dye was $1.0 \mu \mathrm{g} \mathrm{mL}^{-1}$. (b) A typical stacking/ separation process. (c) Band intensity as a function of initial concentration ranging from $0.25 \mu \mathrm{g} \mathrm{mL}^{-1}$ to $4.0 \mu \mathrm{g} \mathrm{mL}^{-1}$. (d) Corresponding images of stacking bands with different initial concentrations at $180 \mathrm{~s}$. Error bars in (a) and (c) represent standard deviation for five independent measurements. The other conditions were the same as those in Fig. 2. brilliant blue was $0.25-4.0 \mu \mathrm{g} \mathrm{mL} \mathrm{m}^{-1}$ (linear regression equation: $y=71.62 \lg x-149.3, y$ for the band intensity, $x$ for brilliant blue at the concentration of $\mathrm{ng} \mathrm{mL}^{-1}$, correlation coefficient: 0.9961). The linear dynamic range for amaranth was $0.25-2.0 \mu \mathrm{g}$ $\mathrm{mL}^{-1}$ (linear regression equation: $y=28.1 x+1.82, y$ for the band intensity, $x$ for amaranth at the concentration of $\mu \mathrm{g} \mathrm{mL} \mathrm{m}^{-1}$, correlation coefficient: 0.9980$)$. The limits of detection $(3 \sigma)$ for amaranth and brilliant blue were $0.31 \mu \mathrm{g} \mathrm{mL} L^{-1}$ and $0.17 \mu \mathrm{g}$ $\mathrm{mL}^{-1}$, respectively. This result indicated that limit of detection of sub ppm level can be achieved colorimetrically with this simple paper device. Again, it should be noted that, in Fig. 4c, each data point was the average result over five independent measurements, and all the RSDs were below 7.5\%. This precision was relatively high considering the simple setup and manual operation. The images of the stacking bands with different initial concentrations from 0.25 to $4.0 \mu \mathrm{g} \mathrm{mL}{ }^{-1}$ are shown in Fig. 4d.

\section{Sample consumption}

In above experiments, we loaded $150 \mu \mathrm{L}$ sample solution to the sample reservoir. For some valuable biosamples, this large sample volume may not be available. As the paper fluidic channel is open, it is easy to reduce the sample consumption by direct loading the sample solution onto the channel. To be specific, $150 \mu \mathrm{L}$ deionized water instead of sample solution was added to the cathode reservoir. After $60 \mathrm{~s}$ establishment of the electric field gradient, $5 \mu \mathrm{L}$ sample solution was loaded onto the paper channel close to the cathode reservoir with pipette. As shown in Fig. S4 (ESI $\dagger$ ), the stacking bands develop quickly after $5 \mathrm{~s}$ of sample loading. The two bands are eventually separated within $30 \mathrm{~s}$.

\section{Simultaneous stacking and separation of proteins}

Simultaneous stacking and separation of proteins in the paper fluidic channel is even more attractive either for sensitive online detection or for further analysis by sophisticated instruments. To demonstrate the feasibility, two colored proteins, bovine hemoglobin $(\mathrm{BHb})$ and cytochrome $\mathrm{c}$ (Cyt c) were tried with the proposed method. Fig. 5a depicts the stacking process for the two model proteins in which the $150 \mu \mathrm{L}$ sample solution was loaded in the cathode reservoir. The initial concentration for BHb was $0.1 \mathrm{mg} \mathrm{mL}^{-1}$ and for Cyt $\mathrm{c}$ it was $0.2 \mathrm{mg} \mathrm{mL}^{-1}$. With application of voltage, two stacking bands were well stacked and separated in the channel within $60 \mathrm{~s}$, and the SR was 1.1 at $t=$ $300 \mathrm{~s}$. Note that the model proteins were prepared in $200 \mathrm{mM}$ Tris solution with a $\mathrm{pH}$ of 10.7 , and $20 \mathrm{mM} \mathrm{NH}_{4} \mathrm{Cl}-\mathrm{NH}_{3}$ with the $\mathrm{pH}$ of 10.8 was taken as the BGE. Both the $\mathrm{pH}$ of sample media and BGE are greater than the pIs of the proteins to make them negatively charged. The conductivity of $20 \mathrm{mM} \mathrm{NH}_{4} \mathrm{Cl}-\mathrm{NH}_{3}$ was $2.80 \mathrm{mS} \mathrm{cm}{ }^{-1}$, which is close to that of $50 \mathrm{mM}$ Tris- $\mathrm{HCl}$ $\left(2.75 \mathrm{mS} \mathrm{cm}^{-1}\right)$. Whereas the conductivity of $200 \mathrm{mM}$ Tris was only $0.11 \mathrm{mS} \mathrm{cm}^{-1}$, much lower than that of the BGE, meeting the basic requirement of FASS. A low sample volume of $5 \mu \mathrm{L}$ was also implemented as aforementioned. Note that $200 \mathrm{mM}$ Tris instead of water was added to cathode reservoir here. As shown in Fig. 5b, the two proteins were stacked within $10 \mathrm{~s}$, and they 


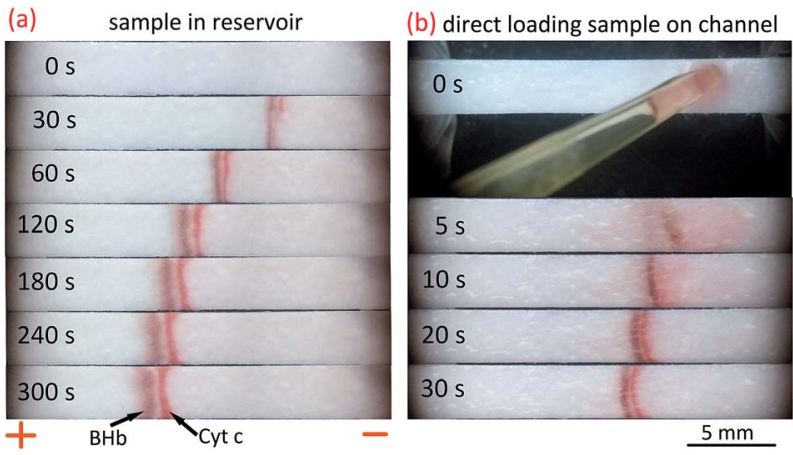

Fig. 5 Paper-based FASS for simultaneous concentration and separation of proteins. (a) A stacking process in which the protein sample $(150 \mu \mathrm{L})$ was added to the cathode reservoir. The initial concentrations for $\mathrm{BHb}$ and Cyt $\mathrm{c}$ were $0.1 \mathrm{mg} \mathrm{mL}^{-1}$ and $0.2 \mathrm{mg} \mathrm{mL}^{-1}$, respectively. (b) Demonstration of small volume protein sample stacking process by loading $5 \mu \mathrm{L}$ sample onto the paper channel instead of the reservoir. The initial concentration for each protein was $2.5 \mathrm{mg} \mathrm{mL}^{-1}$. The proteins were prepared with $200 \mathrm{mM}$ Tris $(\mathrm{pH}=10.7) .20 \mathrm{mM} \mathrm{NH}_{4} \mathrm{Cl}-$ $\mathrm{NH}_{3}(\mathrm{pH}=10.8)$ with $0.4 \%$ HEC was used as the BGE. The other conditions were the same as those in Fig. 2.

were separated at $30 \mathrm{~s}$ with a SR of 0.9 . Here the initial concentration for each protein was $2.5 \mathrm{mg} \mathrm{mL}^{-1}$ for clear observation of the effect.

\section{Conclusions}

In this work, we successfully demonstrated simultaneous sample concentration and separation on a simple straight paper fluidic channel by FASS. This method is low cost and rapid, which is applicable to both small molecule food dyes and macromolecule proteins. This work shows the potential of paper-based FASS on the development of sensitive and multifunctional $\mu$ PADs. A portable power supply together with a smartphone-based imaging system makes this device suitable for on-site analysis. The proposed method may also find its application in protein analysis coupled with other advanced analytical devices such as liquid chromatography or mass spectrometry as a rapid and low cost offline sample pretreatment method.

\section{Acknowledgements}

This research was financially supported by National Natural Science Foundation of China (21575019, 51376039) and Education Department of Liaoning Province of China (LZ2015036).

\section{References}

1 R. Pelton, TrAC, Trends Anal. Chem., 2009, 28, 925-942.

2 A. W. Martinez, S. T. Phillips, G. M. Whitesides and E. Carrilho, Anal. Chem., 2010, 82, 3-10.

3 A. W. Martinez, S. T. Phillips, M. J. Butte and G. M. Whitesides, Angew. Chem., Int. Ed. Engl., 2007, 46, 1318-1320.
4 X. Li, D. R. Ballerini and W. Shen, Biomicrofluidics, 2012, 6, 11301-1130113.

5 M. Santhiago, E. W. Nery, G. P. Santos and L. T. Kubota, Bioanalysis, 2014, 6, 89-106.

6 D. M. Cate, J. A. Adkins, J. Mettakoonpitak and C. S. Henry, Anal. Chem., 2015, 87, 19-41.

7 A. M. Lopez-Marzo and A. Merkoci, Lab Chip, 2016, 16, 31503176.

8 N. A. Meredith, C. Quinn, D. M. Cate, T. H. Reilly 3rd, J. Volckens and C. S. Henry, Analyst, 2016, 141, 1874-1887.

9 C. Zhao and X. Liu, Biomicrofluidics, 2016, 10, 024119.

10 S. T. Sanjay, M. Dou, J. Sun and X. Li, Sci Rep., 2016, 6, 30474. 11 C. Sicard, C. Glen, B. Aubie, D. Wallace, S. JahanshahiAnbuhi, K. Pennings, G. T. Daigger, R. Pelton, J. D. Brennan and C. D. Filipe, Water Res., 2015, 70, 360-369.

12 Y. Zhang, P. Zuo and B. C. Ye, Biosens. Bioelectron., 2015, 68, 14-19.

13 M. Su, L. Ge, S. Ge, N. Li, J. Yu, M. Yan and J. Huang, Anal. Chim. Acta, 2014, 847, 1-9.

14 S. Y. Wong, M. Cabodi, J. Rolland and C. M. Klapperich, Anal. Chem., 2014, 86, 11981-11985.

15 R. Tang, H. Yang, J. R. Choi, Y. Gong, J. Hu, S. Feng, B. Pingguan-Murphy, Q. Mei and F. Xu, Talanta, 2016, 152, 269-276.

16 S. A. Byrnes, J. D. Bishop, L. Lafleur, J. R. Buser, B. Lutz and P. Yager, Lab Chip, 2015, 15, 2647-2659.

17 A. Abbas, A. Brimer, J. M. Slocik, L. Tian, R. R. Naik and S. Singamaneni, Anal. Chem., 2013, 85, 3977-3983.

18 M. M. Gong, P. Zhang, B. D. MacDonald and D. Sinton, Anal. Chem., 2014, 86, 8090-8097.

19 M. M. Gong, R. Nosrati, M. C. San Gabriel, A. Zini and D. Sinton, J. Am. Chem. Soc., 2015, 137, 13913-13919.

20 R. J. Yang, H. H. Pu and H. L. Wang, Biomicrofluidics, 2015, 9, 014122.

21 S. I. Han, K. S. Hwang, R. Kwak and J. H. Lee, Lab Chip, 2016, 16, 2219-2227.

22 S. Hong, R. Kwak and W. Kim, Anal. Chem., 2016, 88, 16821687.

23 L.-H. Hung, H.-L. Wang and R.-J. Yang, Microfluid. Nanofluid., 2016, 20, 1-9.

24 D.-T. Phan, S. A. M. Shaegh, C. Yang and N.-T. Nguyen, Sens. Actuators, B, 2016, 222, 735-740.

25 S. H. Yeh, K. H. Chou and R. J. Yang, Lab Chip, 2016, 16, 925931.

26 B. Y. Moghadam, K. T. Connelly and J. D. Posner, Anal. Chem., 2014, 86, 5829-5837.

27 T. Rosenfeld and M. Bercovici, Lab Chip, 2014, 14, 44654474.

28 X. Li, L. Luo and R. M. Crooks, Lab Chip, 2015, 15, 40904098.

29 B. Y. Moghadam, K. T. Connelly and J. D. Posner, Anal. Chem., 2015, 87, 1009-1017.

30 B. Ma, Y. Z. Song, J. C. Niu and Z. Y. Wu, Lab Chip, 2016, 16, 3460-3465.

31 L. Ge, S. Wang, S. Ge, J. Yu, M. Yan, N. Li and J. Huang, Chem. Commun., 2014, 50, 5699-5702. 
32 C. Xu, M. Zhong, L. Cai, Q. Zheng and X. Zhang, Electrophoresis, 2016, 37, 476-481.

33 L. OuYang, C. Wang, F. Du, T. Zheng and H. Liang, RSC Adv., 2014, 4, 1093-1101.

34 L. Luo, X. Li and R. M. Crooks, Anal. Chem., 2014, 86, 1239012397.

35 C. L. Chagas, F. R. de Souza, T. M. Cardoso, R. C. Moreira, J. A. da Silva, D. P. de Jesus and W. K. Coltro, Anal. Methods, 2016, 8, 6682-6686.

36 H. Cui, K. Horiuchi, P. Dutta and C. F. Ivory, Anal. Chem., 2005, 77, 1303-1309.

37 W. Ouyang, S. H. Ko, D. Wu, A. Y. Wang, P. W. Barone, W. S. Hancock and J. Han, Anal. Chem., 2016, 88, 9669-9677. 38 C. Eid, G. Garcia-Schwarz and J. G. Santiago, Analyst, 2013, 138, 3117-3120.

39 D. R. Laws, D. Hlushkou, R. K. Perdue, U. Tallarek and R. M. Crooks, Anal. Chem., 2009, 81, 8923-8929.
40 D. W. Inglis, E. M. Goldys and N. P. Calander, Angew. Chem., Int. Ed. Engl., 2011, 50, 7546-7550.

41 R. Nosrati, M. M. Gong, M. C. San Gabriel, A. Zini and D. Sinton, Anal. Methods, 2016, 8, 6260-6264.

42 D.-S. Lian, S.-J. Zhao, J. Li and B.-L. Li, Anal. Bioanal. Chem., 2014, 406, 6129-6150.

43 Y. Y. Tu, M. M. Hsieh and S. Y. Chang, Electrophoresis, 2015, 36, 2828-2836.

44 B. Jung, R. Bharadwaj and J. G. Santiago, Electrophoresis, 2003, 24, 3476-3483.

45 K. S. Lee, M. J. Shiddiky, S. H. Park, D. S. Park and Y. B. Shim, Electrophoresis, 2008, 29, 1910-1917.

46 Y. Oyama, T. Osaki, K. Kamiya, R. Kawano, T. Honjoh, H. Shibata, T. Ide and S. Takeuchi, Lab Chip, 2012, 12, 5155-5159.

47 D. Belder and M. Ludwig, Electrophoresis, 2003, 24, 35953606. 